\title{
Initial-Boundary Value Problem for Fractional Partial Differential Equations of Higher Order
}

\author{
Djumaklych Amanov' and Allaberen Ashyralyev²,3 \\ ${ }^{1}$ Institute of Mathematics and Information Technologies, Uzbek Academy of Sciences, \\ 29 Do'rmon yo'li street, Tashkent 100047, Uzbekistan \\ ${ }^{2}$ Department of Mathematics, Fatih University, 34500 Buyucekmece, Istanbul, Turkey \\ ${ }^{3}$ ITTU, Ashgabat 74400, Turkmenistan \\ Correspondence should be addressed to Djumaklych Amanov, damanov@yandex.ru \\ Received 30 March 2012; Revised 25 April 2012; Accepted 12 May 2012 \\ Academic Editor: Valery Covachev
}

Copyright (C) 2012 D. Amanov and A. Ashyralyev. This is an open access article distributed under the Creative Commons Attribution License, which permits unrestricted use, distribution, and reproduction in any medium, provided the original work is properly cited.

The initial-boundary value problem for partial differential equations of higher-order involving the Caputo fractional derivative is studied. Theorems on existence and uniqueness of a solution and its continuous dependence on the initial data and on the right-hand side of the equation are established.

\section{Introduction}

Many problems in viscoelasticity [1-3], dynamical processes in self-similar structures [4], biosciences [5], signal processing [6], system control theory [7], electrochemistry [8], diffusion processes [9], and linear time-invariant systems of any order with internal point delays [10] lead to differential equations of fractional order. For more details of fractional calculus, see [11-15].

The study of existence and uniqueness, periodicity, asymptotic behavior, stability, and methods of analytic and numerical solutions of fractional differential equations have been studied extensively in a large cycle works (see, e.g., [16-42] and the references therein).

In the paper [43], Cauchy problem in a half-space $\left\{(x, y, t):(x, y) \in \mathbb{R}^{2}, t>0\right\}$ for partial pseudodifferential equations involving the Caputo fractional derivative was studied. The existence and uniqueness of a solution and its continuous dependence on the initial data and on the right-hand side of the equation were established.

In the paper [44], the initial-boundary value problem for heat conduction equation with the Caputo fractional derivative was studied. Moreover, in [45], the initial-boundary 
value problem for partial differential equations of higher order with the Caputo fractional derivative was studied in the case when the order of the fractional derivative belongs to the interval $(0,1)$.

In the paper [46], the initial-boundary value problem in plane domain for partial differential equations of fourth order with the fractional derivative in the sense of Caputo was studied in the case when the order of fractional derivative belongs to the interval $(1,2)$. The present paper generalizes results of [46] in the case of space domain for partial differential equations of higher order with a fractional derivative in the sense of Caputo.

The organization of this paper is as follows. In Section 2, we provide the necessary background and formulation of problem. In Section 3, the formal solution of problem is presented. In Sections 4 and 5, the solvability and the regular solvability of the problem are studied. Theorems on existence and uniqueness of a solution and its continuous dependence on the initial data and on the right-hand side of the equation are established. Finally, Section 6 is conclusion.

\section{Preliminaries}

In this section, we present some basic definitions and preliminary facts which are used throughout the paper.

Definition 2.1. If $g(t) \in C[a, b]$ and $\alpha>0$, then the Riemann-Liouville fractional integral is defined by

$$
I_{a+}^{\alpha} g(t)=\frac{1}{\Gamma(\alpha)} \int_{a}^{t} \frac{g(s)}{(t-s)^{1-\alpha}} d s
$$

where $\Gamma(\cdot)$ is the Gamma function defined for any complex number $z$ as

$$
\Gamma(z)=\int_{0}^{\infty} t^{z-1} e^{-t} d t
$$

Definition 2.2. The Caputo fractional derivative of order $\alpha>0$ of a continuous function $g$ : $(a, b) \rightarrow R$ is defined by

$$
{ }^{c} D_{a+}^{\alpha} g(t)=\frac{1}{\Gamma(n-\alpha)} \int_{a}^{t} \frac{g^{(n)}(s)}{(t-s)^{\alpha-n+1}} d s,
$$

where $n=[\alpha]+1$, (the notation $[\alpha]$ stands for the largest integer not greater than $\alpha)$.

Lemma 2.3 (see [13]). Let $p, q \geq 0, f(t) \in L_{1}[0, T]$. Then,

$$
I_{0+}^{p} I_{0+}^{q} f(t)=I_{0+}^{p+q} f(t)=I_{0+}^{q} I_{0+}^{p} f(t)
$$

is satisfied almost everywhere on $[0, T]$. Moreover, if $f(t) \in C[0, T]$, then (2.4) is true and ${ }^{c} D_{0+}^{\alpha} I_{0+}^{\alpha} f(t)=f(t)$ for all $t \in[0, T]$ and $\alpha>0$. 
Theorem 2.4 (see [47, page 123]). Let $f(t) \in L_{1}(0, T)$. Then, the integral equation

$$
z(t)=f(t)+\lambda \int_{0}^{t} \frac{(t-\tau)^{\alpha-1}}{\Gamma(\alpha)} z(\tau) d \tau
$$

has a unique solution $z(t)$ defined by the following formula:

$$
z(t)=f(t)+\lambda \int_{0}^{t}(t-\tau)^{\alpha-1} E_{\alpha, \alpha}\left(\lambda(t-\tau)^{\alpha}\right) f(\tau) d \tau
$$

where $E_{\alpha, \beta}(z)=\sum_{k=0}^{\infty}\left(z^{k} / \Gamma(k \alpha+\beta)\right)$ is a Mittag-Leffler type function.

For the convenience of the reader, we give the proof of Theorem 2.4, applying the fixed-point iteration method. We denote

$$
B z(t)=\lambda \int_{0}^{t} \frac{(t-\tau)^{\alpha-1}}{\Gamma(\alpha)} z(\tau) d \tau
$$

Then,

$$
z(t)=\sum_{k=0}^{m-1} B^{k} f(t)+B^{m} z(t), \quad m=1, \ldots, n
$$

The proof of this theorem is based on formula (2.8) and

$$
B^{m} z(t)=\lambda^{m} \int_{0}^{t} \frac{(t-\tau)^{m \alpha-1}}{\Gamma(m \alpha)} z(\tau) d \tau
$$

for any $m \in N$. Let us prove (2.9) for any $m \in N$. For $m=1$, it follows from (2.7) directly. Assume that (2.9) holds for some $m-1 \in N$. Then, applying (2.7) and (2.9) for $m-1 \in N$, we get

$$
\begin{aligned}
B^{m} z(t) & =\lambda^{m-1} \int_{0}^{t} \frac{(t-s)^{(m-1) \alpha-1}}{\Gamma((m-1) \alpha)} B z(s) d s \\
& =\lambda^{m-1} \int_{0}^{t} \frac{(t-s)^{(m-1) \alpha-1}}{\Gamma((m-1) \alpha)} \lambda \int_{0}^{s}(s-\tau)^{\alpha-1} z(\tau) d \tau d s \\
& =\frac{\lambda^{m}}{\Gamma(\alpha) \Gamma((m-1) \alpha)} \int_{0}^{t} \int_{0}^{s}(t-s)^{(m-1) \alpha-1}(s-\tau)^{\alpha-1} z(\tau) d \tau d s \\
& =\frac{\lambda^{m}}{\Gamma(\alpha) \Gamma((m-1) \alpha)} \int_{0}^{t} \int_{\tau}^{t}(t-s)^{(m-1) \alpha-1}(s-\tau)^{\alpha-1} d s z(\tau) d \tau
\end{aligned}
$$


Performing the change of variables $s-\tau=(t-\tau) p$, we get

$$
\begin{aligned}
\int_{\tau}^{t}(t-s)^{(m-1) \alpha-1}(s-\tau)^{\alpha-1} d s & =(t-\tau)^{m \alpha-1} \int_{0}^{1}(1-p)^{(m-1) \alpha-1} p^{\alpha-1} d p \\
& =(t-\tau)^{m \alpha-1} B((m-1) \alpha, \alpha) \\
& =\frac{(t-\tau)^{m \alpha-1}}{\Gamma(m \alpha)} \Gamma((m-1) \alpha) \Gamma(\alpha) .
\end{aligned}
$$

Then,

$$
B^{m} z(t)=\lambda^{m} \int_{0}^{t} \frac{(t-\tau)^{m \alpha-1}}{\Gamma(m \alpha)} z(\tau) d \tau
$$

So, identity (2.9) holds for $m \in N$. Therefore, by induction identity (2.9) holds for any $m \in N$. In the space domain, $\Omega=\{(x, y, t): 0<x<p, 0<y<q, 0<t<T\}$, we consider the initial-boundary value problem:

$$
\begin{aligned}
& (-1)^{k_{c}} D_{0+}^{\alpha} u+\frac{\partial^{2 k} u}{\partial x^{2 k}}+\frac{\partial^{2 k} u}{\partial y^{2 k}}=f(x, y, t), \quad 0<x<p, 0<y<q, 0<t<T, \\
& \frac{\partial^{2 m} u(0, y, t)}{\partial x^{2 m}}=\frac{\partial^{2 m} u(p, y, t)}{\partial x^{2 m}}=0, \quad m=0,1, \ldots, k-1,0 \leq y \leq q, 0 \leq t \leq T, \\
& \frac{\partial^{2 m} u(x, 0, t)}{\partial y^{2 m}}=\frac{\partial^{2 m} u(x, q, t)}{\partial y^{2 m}}=0, \quad m=0,1, \ldots, k-1,0 \leq x \leq p, 0 \leq t \leq T, \\
& u(x, y, 0)=\varphi(x, y), \quad u_{t}(x, y, 0)=\psi(x, y), \quad 0 \leq x \leq p, 0 \leq y \leq q
\end{aligned}
$$

for partial differential equations of higher order with the fractional derivative order $\alpha \in(1,2)$ in the sense of Caputo. Here, $k(k \geq 1)$ is a fixed positive integer number.

\section{The Construction of the Formal Solution of (2.13)}

We seek a solution of problem (2.13) in the form of Fourier series:

$$
u(x, y, t)=\sum_{n, m=1}^{\infty} u_{n m}(t) v_{n m}(x, y)
$$

expanded along a complete orthonormal system:

$$
v_{n m}(x, y)=\frac{2}{\sqrt{p q}} \sin \frac{n \pi}{p} x \sin \frac{m \pi}{q} y, \quad 1 \leq n, m<\infty .
$$


We denote

$$
\begin{aligned}
& \Omega_{0}=\bar{\Omega} \cap(t=0)=\{(x, y, 0): 0 \leq x \leq p, 0 \leq y \leq q\}, \\
& \frac{n \pi}{p}=v_{n}, \quad \frac{m \pi}{q}=\mu_{m}, \quad v_{n}^{2 k}+\mu_{m}^{2 k}=\lambda_{n m}^{2 k}, \quad 1 \leq n, m<\infty .
\end{aligned}
$$

We expand the given function $f(x, y, t)$ in the form of a Fourier series along the functions $v_{n m}(x, y), 1 \leq n, m<\infty$ :

$$
f(x, y, t)=\sum_{n, m=1}^{\infty} f_{n m}(t) v_{n m}(x, y)
$$

where

$$
f_{n m}(\mathrm{t})=\int_{0}^{p} \int_{0}^{q} f(x, y, t) v_{n m}(x, y) d y d x, \quad 1 \leq n, m<\infty
$$

Substituting (3.1) and (3.4) into (2.13), we obtain

$$
(-1)^{k}{ }^{c} D_{0+}^{\alpha} u_{n m}(t)+(-1)^{k} \lambda_{n m}^{2 k} u_{n m}(t)=f_{n m}(t) .
$$

By Lemma 2.3, we have that

$$
{ }^{c} D_{0+}^{\alpha} u_{n m}(t)=I_{0+}^{2-\alpha} u_{n m}^{\prime \prime}(t),
$$

where

$$
I_{0+}^{\alpha} f(t)=\frac{1}{\Gamma(\alpha)} \int_{0}^{t}(t-\tau)^{\alpha-1} f(\tau) d \tau
$$

is Riemann-Liouville integral of fractional order $\alpha$. Using (3.6) and (3.7), we get the following equation:

$$
I_{0+}^{2-\alpha} u_{n m}^{\prime \prime}(t)+\lambda_{n m}^{2 k} u_{n m}(t)=(-1)^{k} f_{n m}(t)
$$

Applying the operator $I_{0+}^{\alpha}$ to this equation, we get the following Volterra integral equation of the second kind:

$$
u_{n m}(t)=\frac{-\lambda_{n m}^{2 k}}{\Gamma(\alpha)} \int_{0}^{t}(t-\tau)^{\alpha-1} u_{n m}(\tau) d \tau+u_{n m}(0)+t u_{n m}^{\prime}(0)+(-1)^{k} I_{0+}^{\alpha} f_{n m}(t)
$$


According to the Theorem 2.4, (3.10) has a unique solution $u_{n m}(t)$ defined by the following formula:

$$
\begin{aligned}
u_{n m}(t)= & \frac{(-1)^{k}}{\Gamma(\alpha)} \int_{0}^{t}(t-\tau)^{\alpha-1} f_{n m}(\tau) d \tau \\
& +u_{n m}(0)\left[1-\lambda_{n m}^{2 k} \int_{0}^{t}(t-\tau)^{\alpha-1} E_{\alpha, \alpha}\left(-\lambda_{n m}^{2 k}(t-\tau)^{\alpha}\right) d \tau\right] \\
& +u_{n m}^{\prime}(0)\left[t-\lambda_{n m}^{2 k} \int_{0}^{t}(t-\tau)^{\alpha-1} E_{\alpha, \alpha}\left(-\lambda_{n m}^{2 k}(t-\tau)^{\alpha}\right) \tau d \tau\right] \\
& -\frac{\lambda_{n m}^{2 k}}{\Gamma(\alpha)} \int_{0}^{t}(t-\eta)^{\alpha-1} E_{\alpha, \alpha}\left(-\lambda_{n m}^{2 k}(t-\eta)^{\alpha}\right) d \eta \int_{0}^{\eta}(\eta-\tau)^{\alpha-1} f_{n m}(\tau) d \tau
\end{aligned}
$$

Using the formula (see, e.g., [27, page 118] and [47, page 120])

$$
\begin{gathered}
\frac{1}{\Gamma(\beta)} \int_{0}^{z} t^{\mu-1} E_{\alpha, \mu}\left(\lambda t^{\alpha}\right)(z-t)^{\beta-1} d t=z^{\mu+\beta-1} E_{\alpha, \mu+\beta}\left(\lambda z^{\alpha}\right), \\
\frac{1}{\Gamma(\mu)}+z E_{\alpha, \alpha+\mu}(z)=E_{\alpha, \mu}(z),
\end{gathered}
$$

we get

$$
\begin{aligned}
- & \frac{\lambda_{n m}^{2 k}}{\Gamma(\alpha)} \int_{0}^{t}(t-\eta)^{\alpha-1} E_{\alpha, \alpha}\left(-\lambda_{n m}^{2 k}(t-\eta)^{\alpha}\right) d \eta \int_{0}^{\eta}(\eta-\tau)^{\alpha-1} f_{n m}(\tau) d \tau \\
= & \int_{0}^{t} f_{n m}(\tau)\left\{-\frac{\lambda_{n m}^{2 k}}{\Gamma(\alpha)} \int_{\tau}^{t}(t-\eta)^{\alpha-1} E_{\alpha, \alpha}\left(-\lambda_{n m}^{2 k}(t-\eta)^{\alpha}\right)(\eta-\tau)^{\alpha-1} d \eta\right\} d \tau \\
= & \int_{0}^{t} f_{n m}(\tau)\left\{-\frac{\lambda_{n m}^{2 k}}{\Gamma(\alpha)} \int_{0}^{t-\tau} z^{\alpha-1} E_{\alpha, \alpha}\left(-\lambda_{n m}^{2 k} z^{\alpha}\right)(t-\tau-z)^{\alpha-1} d z\right\} d \tau \\
= & -\int_{0}^{t} f_{n m}(\tau) \lambda_{n m}^{2 k}(t-\tau)^{2 \alpha-1} E_{\alpha, 2 \alpha}\left(-\lambda_{n m}^{2 k}(t-\tau)^{\alpha}\right) d \tau \\
= & \int_{0}^{t}(t-\tau)^{\alpha-1} f_{n m}(\tau)\left\{-\frac{1}{\Gamma(\alpha)}+E_{\alpha, \alpha}\left(-\lambda_{n m}^{2 k}(t-\tau)^{\alpha}\right)\right\} d \tau \\
& -\lambda_{n m}^{2 k} \int_{0}^{t}(t-\tau)^{\alpha-1} E_{\alpha, \alpha}\left(-\lambda_{n m}^{2 k}(t-\tau)^{\alpha}\right) d \tau \\
= & -\lambda_{n m}^{2 k} \int_{0}^{t} z^{\alpha-1} E_{\alpha, \alpha}\left(-\lambda_{n m}^{2 k} z^{\alpha}\right)(t-z)^{1-1} d z
\end{aligned}
$$


Abstract and Applied Analysis

$$
\begin{aligned}
= & \Gamma(1) \lambda_{n m}^{2 k} t^{\alpha} E_{\alpha, \alpha+1}\left(-\lambda_{n m}^{2 k} t^{\alpha}\right)=E_{\alpha, 1}\left(-\lambda_{n m}^{2 k} t^{\alpha}\right)-1, \\
& -\lambda_{n m}^{2 k} \int_{0}^{t}(t-\tau)^{\alpha-1} E_{\alpha, \alpha}\left(-\lambda_{n m}^{2 k}(t-\tau)^{\alpha}\right) \tau d \tau \\
= & -\lambda_{n m}^{2 k} \int_{0}^{t} z^{\alpha-1} E_{\alpha, \alpha}\left(-\lambda_{n m}^{2 k} z^{\alpha}\right)(t-z)^{2-1} d z \\
= & \Gamma(2) \lambda_{n m}^{2 k} t^{\alpha+1} E_{\alpha, \alpha+2}\left(-\lambda_{n m}^{2 k} t^{\alpha}\right)=t E_{\alpha, 2}\left(-\lambda_{n m}^{2 k} t^{\alpha}\right)-t .
\end{aligned}
$$

From these three formulas and (3.11), it follows that

$$
\begin{aligned}
u_{n m}(t)= & u_{n m}(0) E_{\alpha, 1}\left(-\lambda_{n m}^{2 k} t^{\alpha}\right)+t u_{n m}^{\prime}(0) E_{\alpha, 2}\left(-\lambda_{n m}^{2 k} t^{\alpha}\right) \\
& +(-1)^{k} \int_{0}^{t}(t-\tau)^{\alpha-1} E_{\alpha, \alpha}\left(-\lambda_{n m}^{2 k}(t-\tau)^{\alpha}\right) f_{n m}(\tau) d \tau .
\end{aligned}
$$

For $u_{n m}(0)$ and $u_{n m}^{\prime}(0)$, we expand the given functions $\varphi(x, y)$ and $\psi(x, y)$ in the form of a Fourier series along the functions $v_{n m}(x, y), 1 \leq n, m<\infty$ :

$$
\begin{aligned}
& \varphi(x, y)=\sum_{n, m=1}^{\infty} \varphi_{n m} v_{n m}(x, y), \\
& \psi(x, y)=\sum_{n, m=1}^{\infty} \psi_{n m} v_{n m}(x, y),
\end{aligned}
$$

where

$$
\begin{aligned}
\varphi_{n m} & =\int_{0}^{p} \int_{0}^{q} \varphi(x, y) v_{n m}(x, y) d y d x \\
\psi_{n m} & =\int_{0}^{p} \int_{0}^{q} \psi(x, y) v_{n m}(x, y) d y d x
\end{aligned}
$$

Using (2.13), (3.14), (3.16), we obtain

$$
\begin{aligned}
u_{n m}(t)= & E_{\alpha, 1}\left(-\lambda_{n m}^{2 k} t^{\alpha}\right) \varphi_{n m}+t E_{\alpha, 2}\left(-\lambda_{n m}^{2 k} t^{\alpha}\right) \psi_{n m} \\
& +(-1)^{k} \int_{0}^{t}(t-\tau)^{\alpha-1} E_{\alpha, \alpha}\left(-\lambda_{n m}^{2 k}(t-\tau)^{\alpha}\right) f_{n m}(\tau) d \tau .
\end{aligned}
$$

So, the unique solution of (3.10) is defined by (3.17). Consequently, the unique solution of problem (2.13) is defined by (3.1). 
Applying the formula (3.17), the Cauchy-Schwarz inequality, and the estimate (see $[13$, page 136])

$$
\left|E_{\alpha, \beta}(z)\right| \leq \frac{M}{1+|z|}, \quad M=\text { const }>0, \operatorname{Re} z<0,
$$

we get the following inequality:

$$
\left|u_{n m}(t)\right| \leq C_{0}\left(\left|\varphi_{n m}\right|+\left|\psi_{n m}\right|+\left(\int_{0}^{t}\left|f_{n m}(t)\right|^{2} d t\right)^{1 / 2}\right)
$$

for the solution of (3.10) for any $t, t \in[0, T]$. Here, $C_{0}=\max \left\{M, T M, M\left(T^{\alpha-1 / 2} / \sqrt{2 \alpha-1}\right)\right\}$.

\section{Solvability of $(2.13)$ in $L_{2}(\Omega)$ Space}

Now, we will prove that the solution $u(x, y, t)$ of problem (2.13) continuously depends on $\varphi(x, y), \psi(x, y)$, and $f(x, y, t)$.

Theorem 4.1. Suppose $\varphi(x, y) \in L_{2}\left(\Omega_{0}\right), \psi(x, y) \in L_{2}\left(\Omega_{0}\right)$, and $f(x, y, t) \in L_{2}(\Omega)$, then the series (3.1) converges in $L_{2}(\Omega)$ to $u \in L_{2}(\Omega)$ and for the solution of problem (2.13), the following stability inequality

$$
\|u\|_{L_{2}(\Omega)} \leq C_{1}\left(\|\varphi\|_{L_{2}\left(\Omega_{0}\right)}+\|\psi\|_{L_{2}\left(\Omega_{0}\right)}+\|f\|_{L_{2}(\Omega)}\right)
$$

holds, where $C_{1}$ does not depend on $\varphi(x, y), \psi(x, y)$, and $f(x, y, t)$.

Proof. We consider the sum:

$$
u_{N}(x, y, t)=\sum_{n, m=1}^{N} u_{n m}(t) v_{n m}(x, y)
$$

where $N$ is a natural number. For the positive integer number $L$, we have that

$$
\begin{aligned}
\left\|u_{N+L}-u_{N}\right\|_{L_{2}(\Omega)}^{2} & =\left\|\sum_{n, m=N+1}^{N+L} u_{n m}(\cdot) v_{n m}(\cdot, \cdot)\right\|_{L_{2}(\Omega)}^{2} \\
& =\sum_{n, m=N+1}^{N+L} \int_{0}^{T}\left|u_{n m}(t)\right|^{2} d t .
\end{aligned}
$$


Applying (3.19), we get

$$
\begin{aligned}
\sum_{n, m=1}^{\infty} \int_{0}^{T}\left|u_{n m}(t)\right|^{2} d t & \leq 3 C_{0}^{2}\left(\sum_{n, m=1}^{\infty}\left|\varphi_{n m}\right|^{2}+\sum_{n, m=1}^{\infty}\left|\psi_{n m}\right|^{2}+\sum_{n, m=1}^{\infty} \int_{0}^{T}\left|f_{n m}(t)\right|^{2} d t\right) \\
& =C^{2}\left(\|\varphi\|_{L_{2}\left(\Omega_{0}\right)}^{2}+\|\psi\|_{L_{2}\left(\Omega_{0}\right)}^{2}+\|f\|_{L_{2}(\Omega)}^{2}\right),
\end{aligned}
$$

where $C^{2}=3 T C_{0}^{2}$. Therefore, $\sum_{n, m=N+1}^{N+L} \int_{0}^{T}\left|u_{n m}(t)\right|^{2} d t \rightarrow 0$ as $N \rightarrow \infty$. Consequently, the series (3.1) converges in $L_{2}(\Omega)$ to $u(x, y, t) \in L_{2}(\Omega)$. Inequality (4.1) for the solution of problem (2.13) follows from the estimate (4.4). Theorem 4.1 is proved.

\section{The Regular Solvability of (2.13)}

In this section, we will study theregular solvability of problem (2.13).

Lemma 5.1. Suppose $\varphi(x, y) \in C^{1}\left(\bar{\Omega}_{0}\right), \varphi_{x y}(x, y) \in L_{2}\left(\Omega_{0}\right), \psi(x, y) \in C^{1}\left(\bar{\Omega}_{0}\right), \psi_{x y}(x, y) \in$ $L_{2}\left(\Omega_{0}\right), \varphi(x, y)=0$ on $\partial \Omega_{0}, \psi(x, y)=0$ on $\partial \Omega_{0}, f(x, y, t) \in C^{2}(\bar{\Omega}), f_{x x y}(x, y, t) \in$ $C(\bar{\Omega}) f_{x y y}(x, y, t) \in C\left(\bar{\Omega}_{0}\right), f_{x x y y}(x, y, t) \in C\left(\bar{\Omega}_{0}\right)$, and $f(x, y, t)=0$ on $\partial \Omega \times[0, T]$. Then, for any $\varepsilon \in(0,1)$, the following estimates

$$
\begin{gathered}
\left|u_{n m}(t)\right| \leq C_{1}\left(\frac{\left|\varphi_{n m}\right|}{v_{n}^{k} \mu_{m}^{k}}+\frac{\left|\psi_{n m}\right|}{v_{n}^{k} \mu_{m}^{k}}+\frac{1}{v_{n}^{k+1} \mu_{m}^{k+1}}+\frac{1}{v_{n}^{k+1-\varepsilon} \mu_{m}^{k+1}}+\frac{1}{v_{n}^{k+1} \mu_{m}^{k+1-\varepsilon}}\right), \\
\lambda_{n m}^{2 k}\left|u_{n m}(t)\right| \leq C_{2}\left(\frac{\left|\varphi_{n m}^{(1,1)}\right|}{v_{n} \mu_{m}}+\frac{\left|\psi_{n m}^{(1,1)}\right|}{v_{n} \mu_{m}}+\frac{1}{v_{n}^{2} \mu_{m}^{2}}+\frac{1}{v_{n}^{2-\varepsilon} \mu_{m}^{2}}+\frac{1}{v_{n}^{2} \mu_{m}^{2-\varepsilon}}\right)
\end{gathered}
$$

hold, where $C_{1}$ and $C_{2}$ do not depend on $\varphi(x, y)$ and $\psi(x, y)$.

Proof. Integrating by parts with respect to $x$ and $y$ in (3.5), (3.16), we get

$$
\begin{gathered}
\varphi_{n m}=\frac{1}{v_{n} \mu_{m}} \varphi_{n m}^{(1,1)}, \\
\psi_{n m}=\frac{1}{v_{n} \mu_{m}} \psi_{n m}^{(1,1)}, \\
f_{n m}(t)=\frac{1}{v_{n} \mu_{m}} f_{n m}^{(1,1,0)}(t), \\
f_{n m}(t)=\frac{1}{v_{n}^{2} \mu_{m}^{2}} f_{n m}^{(2,2,0)}(t),
\end{gathered}
$$


where

$$
\begin{gathered}
\varphi_{n m}^{(1,1)}=\int_{0}^{p} \int_{0}^{q} \frac{\partial^{2} \varphi(x, y)}{\partial x \partial y} v_{n m}(x, y) d y d x, \\
\psi_{n m}^{(1,1)}=\int_{0}^{p} \int_{0}^{q} \frac{\partial^{2} \psi(x, y)}{\partial x \partial y} v_{n m}(x, y) d y d x, \\
f_{n m}^{(1,1,0)}(t)=\int_{0}^{p} \int_{0}^{q} \frac{\partial^{2} f(x, y, t)}{\partial x \partial y} v_{n m}(x, y) d y d x, \\
f_{n m}^{(2,2,0)}(t)=\int_{0}^{p} \int_{0}^{q} \frac{\partial^{4} f(x, y, t)}{\partial x^{2} \partial y^{2}} v_{n m}(x, y) d y d x
\end{gathered}
$$

Under the assumptions of Lemma 5.1, it follows that the functions $f_{n m}^{(1,1,0)}(t)$ and $f_{n m}^{(2,2,0)}(t)$ are bounded, that is,

$$
\left|f_{n m}^{(1,1,0)}(t)\right| \leq N_{1}, \quad\left|f_{n m}^{(2,2,0)}(t)\right| \leq N_{2}
$$

where $N_{1}=$ const $>0, N_{2}=$ const $>0$. Let $0<t_{0} \leq t \leq T$, where $t_{0}$ is a sufficiently small number. For sufficiently large $n$ and $m$, the following inequalities are true:

$$
\begin{gathered}
\ln \lambda_{n m}^{\varepsilon}<\lambda_{n m}^{\varepsilon}<\nu_{n}^{\varepsilon}+\mu_{m}^{\varepsilon}, \quad 0<\varepsilon<1, \\
1+\lambda_{n m}^{2 k} T^{\alpha}<2 \lambda_{n m}^{2 k} T^{\alpha} .
\end{gathered}
$$

Using (3.16), (5.8), (5.9), and (3.17), we get

$$
\begin{aligned}
\left|u_{n m}(t)\right| & \leq M\left(\frac{1}{2 t_{0}^{\alpha}} \frac{\left|\varphi_{n m}\right|}{v_{n}^{k} \mu_{m}^{k}}+\frac{1}{2 t_{0}^{\alpha-1}} \frac{\left|\varphi_{n m}\right|}{v_{n}^{k} \mu_{m}^{k}}-\frac{N_{1}}{\alpha v_{n}^{k+1} \mu_{m}^{k+1}} \int_{0}^{t} \frac{d\left(1+\lambda_{n m}^{2 k}(t-\tau)^{\alpha}\right)}{1+\lambda_{n m}^{2 k}(t-\tau)^{\alpha}}\right) \\
& \leq M\left(\frac{1}{2 t_{0}^{\alpha}} \frac{\left|\varphi_{n m}\right|}{v_{n}^{k} \mu_{m}^{k}}+\frac{1}{2 t_{0}^{\alpha-1}} \frac{\left|\psi_{n m}\right|}{v_{n}^{k} \mu_{m}^{k}}+\frac{N_{1}\left(\ln 2 T^{\alpha}+(2 k / \varepsilon) \ln \lambda_{n m}^{\varepsilon}\right)}{\alpha v_{n}^{k+1} \mu_{m}^{k+1}}\right) \\
& \leq C_{1}\left(\frac{\left|\varphi_{n m}\right|}{v_{n}^{k} \mu_{m}^{k}}+\frac{\left|\psi_{n m}\right|}{v_{n}^{k} \mu_{m}^{k}}+\frac{1}{v_{n}^{k+1} \mu_{m}^{k+1}}+\frac{1}{v_{n}^{k+1-\varepsilon} \mu_{m}^{k+1}}+\frac{1}{v_{n}^{k+1} \mu_{m}^{k+1-\varepsilon}}\right),
\end{aligned}
$$


where $C_{1}=\max \left\{M / 2 t_{0}^{\alpha}, M / 2 t_{0}^{\alpha-1}, M N_{1} \ln 2 T^{\alpha} / \alpha, 2 k M N_{1} / \alpha \varepsilon\right\}$. Thus, inequality (5.1) is obtained. Now, we will prove inequality (5.2). Using (5.3), (5.4), (5.6), (5.8), (5.9), and (3.17), we get

$$
\begin{aligned}
\lambda_{n m}^{2 k}\left|u_{n m}(t)\right| & \leq M\left(\frac{\left|\varphi_{n m}\right|}{t^{\alpha}}+\frac{\left|\psi_{n m}\right|}{t^{\alpha-1}}+\lambda_{n m}^{2 k} \int_{0}^{t} \frac{(t-\tau)^{\alpha-1} f_{n m}(\tau)}{1+\lambda_{n m}^{2 k}(t-\tau)^{\alpha}} d \tau\right) \\
& \leq M\left(\frac{1}{t_{0}^{\alpha}} \frac{\left|\varphi_{n m}^{(1,1)}\right|}{v_{n} \mu_{m}}+\frac{\left|\psi_{n m}^{(1,1)}\right|}{t_{0}^{\alpha-1} v_{n} \mu_{m}}-\frac{N_{2}}{\alpha} \int_{0}^{t} \frac{d\left(1+\lambda_{n m}^{2 k}(t-\tau)^{\alpha}\right)}{v_{n}^{2} \mu_{m}^{2}\left(1+\lambda_{n m}^{2 k}(t-\tau)^{\alpha}\right)}\right) \\
& \leq \frac{M}{t_{0}^{\alpha}} \frac{\left|\varphi_{n m}^{(1,1)}\right|}{v_{n} \mu_{m}}+\frac{M}{t_{0}^{\alpha-1}} \frac{\left|\psi_{n m}^{(1,1)}\right|}{v_{n} \mu_{m}}+\frac{2 M N_{2} \ln T^{\alpha}}{\alpha v_{n}^{2} \mu_{m}^{2}}+\frac{2 k M N_{2}}{\alpha \varepsilon v_{n}^{2-\varepsilon} \mu_{m}^{2}}+\frac{2 k M N_{2}}{\alpha \varepsilon v_{n}^{2} \mu_{m}^{2-\varepsilon}} \\
& \leq C_{2}\left(\frac{\left|\varphi_{n m}^{(1,1)}\right|}{v_{n} \mu_{m}}+\frac{\left|\psi_{n m}^{(1,1)}\right|}{v_{n} \mu_{m}}+\frac{1}{v_{n}^{2} \mu_{m}^{2}}+\frac{1}{v_{n}^{2-\varepsilon} \mu_{m}^{2}}+\frac{1}{v_{n}^{2} \mu_{m}^{2-\varepsilon}}\right),
\end{aligned}
$$

where $C_{2}=\max \left\{M / t_{0}^{\alpha}, M / t_{0}^{\alpha-1}, M N_{2} \ln 2 T^{\alpha} / \alpha, 2 k M N_{2} / \alpha \varepsilon\right\}$. Lemma 5.1 is proved.

Theorem 5.2. Suppose that the assumptions of Lemma 5.1 hold. Then, there exists a regular solution of problem (2.13).

Proof. We will prove uniform and absolute convergence of series (3.1) and

$$
\begin{gathered}
\frac{\partial^{2 k} u(x, y, t)}{\partial x^{2 k}}=\sum_{n, m=1}^{\infty}(-1)^{k} v_{n}^{2 k} u_{n m}(t) v_{n m}(x, y), \\
\frac{\partial^{2 k} u(x, y, t)}{\partial x^{2 k}}=\sum_{n, m=1}^{\infty}(-1)^{k} \mu_{n}^{2 k} u_{n m}(t) v_{n m}(x, y), \\
{ }^{c} D_{0+}^{\alpha} u(x, y, t)=-\sum_{n, m=1}^{\infty}(-1)^{k} \lambda_{n m}^{2 k} u_{n m}(t) v_{n m}(x, y)+\sum_{n, m=1}^{\infty} f_{n m}(t) v_{n m}(x, y) .
\end{gathered}
$$

The series

$$
\sum_{n, m=1}^{\infty}\left|u_{n m}(t)\right|
$$

is majorant for the series (3.1). From (5.1), it follows that the series (5.15) uniformly converges. Actually,

$$
\sum_{n, m=1}^{\infty}\left|u_{n m}(t)\right| \leq C \sum_{n, m=1}^{\infty}\left(\frac{\left|\varphi_{n m}\right|}{v_{n}^{k} \mu_{m}^{k}}+\frac{\left|\psi_{n m}\right|}{v_{n}^{k} \mu_{m}^{k}}+\frac{1}{v_{n}^{k} \mu_{n}^{k}}+\frac{1}{v_{n}^{k+1-\varepsilon} \mu_{n}^{k+1}}+\frac{1}{v_{n}^{k+1} \mu_{n}^{k+1-\varepsilon}}\right)
$$


Applying the Cauchy-Schwarz inequality and the Parseval equality, we obtain

$$
\begin{aligned}
\sum_{n, m=1}^{\infty} \frac{\left|\varphi_{n m}\right|}{v_{n}^{k} \mu_{m}^{k}} & \leq\left(\sum_{n, m=1}^{\infty} \frac{1}{v_{n}^{2 k} \mu_{m}^{2 k}}\right)^{1 / 2}\left(\sum_{n, m=1}^{\infty}\left|\varphi_{n m}\right|^{2}\right)^{1 / 2} \\
& =\frac{p^{k} q^{k}}{\pi^{2 k}}\left(\sum_{n=1}^{\infty} \frac{1}{n^{2 k}} \sum_{m=1}^{\infty} \frac{1}{m^{2 k}}\right)^{1 / 2}\|\varphi\|_{L_{2}\left(\Omega_{0}\right)} .
\end{aligned}
$$

Analogously, we get

$$
\sum_{n, m=1}^{\infty} \frac{\left|\psi_{n m}\right|}{v_{n}^{k} \mu_{m}^{k}} \leq \frac{p^{k} q^{k}}{\pi^{2 k}}\left(\sum_{n=1}^{\infty} \frac{1}{n^{2 k}} \sum_{m=1}^{\infty} \frac{1}{m^{2 k}}\right)^{1 / 2}\|\psi\|_{L_{2}\left(\Omega_{0}\right)}
$$

Since $2 k \geq 2$, then the series $\sum_{n=1}^{\infty}\left(1 / n^{2 k}\right), \sum_{m=1}^{\infty}\left(1 / m^{2 k}\right)$ converges by the integral test. Further, $k+1-\varepsilon>1$, then the series

$$
\sum_{n, m=1}^{\infty} \frac{1}{v_{n}^{k+1} \mu_{m}^{k+1}}, \quad \sum_{n, m=1}^{\infty} \frac{1}{v_{n}^{k+1-\varepsilon} \mu_{m}^{k+1}}, \quad \sum_{n, m=1}^{\infty} \frac{1}{v_{n}^{k+1} \mu_{m}^{k+1-\varepsilon}}
$$

converges also by the integral test for any $k \geq 1$ and $\varepsilon \in(0,1)$.

Consequently, the series (3.1) absolutely and uniformly converges in the domain $\Omega_{t_{0}}=$ $\Omega \times\left[t_{0}, T\right]$ for any $t_{0} \in(0, T)$. At $t=0$, the series (3.1) converges and has a sum equal to $\varphi(x, y)$. Since $v_{n}^{2 k}<\lambda_{n m}^{2 k}, \mu_{m}^{2 k}<\lambda_{n m}^{2 k}$, then the series

$$
\sum_{n, m=1}^{\infty} \lambda_{n m}^{2 k}\left|u_{n m}\right|
$$

is majorant for the series (5.12), (5.13) and for the first series from (5.14). From (5.2), it follows that the series (5.20) uniformly converges. Indeed, using the Parseval equality and CauchySchwarz inequality, we get

$$
\sum_{n, m=1}^{\infty} \frac{\left|\varphi_{n m}^{(1,1)}\right|}{v_{n} \mu_{m}} \leq\left(\sum_{n=1}^{\infty} \frac{1}{v_{n}^{2}} \sum_{m=1}^{\infty} \frac{1}{m^{2}}\right)^{1 / 2}\left(\sum_{n, m=1}^{\infty}\left|\varphi_{n m}^{(1,1)}\right|^{2}\right)^{1 / 2}=\frac{p q}{6}\left\|\frac{\partial^{2} \varphi}{\partial x \partial y}\right\|_{L_{2}\left(\Omega_{0}\right)}
$$

Analogously, we conclude that

$$
\sum_{n, m=1}^{\infty} \frac{\left|\psi_{n m}^{(1,1)}\right|}{v_{n} \mu_{m}} \leq \frac{p q}{6}\left\|\frac{\partial^{2} \psi}{\partial x \partial y}\right\|_{L_{2}\left(\Omega_{0}\right)}
$$


The series

$$
\sum_{n, m=1}^{\infty}\left(\frac{1}{v_{n}^{2} \mu_{m}^{2}}+\frac{1}{v_{n}^{2-\varepsilon} \mu_{m}^{2}}+\frac{1}{v_{n}^{2} \mu_{m}^{2-\varepsilon}}\right)
$$

converges for any $\varepsilon \in(0,1)$ according to the integral test. The series

$$
\sum_{n, m=1}^{\infty}\left|f_{n m}(t)\right|
$$

is majorant for the second series from (5.14). From (5.6) and (5.8), it follows that the series (5.14) uniformly converges. Indeed,

$$
\sum_{n, m=1}^{\infty}\left|f_{n m}(t)\right|=\sum_{n, m=1}^{\infty} \frac{1}{v_{n}^{2} \mu_{m}^{2}}\left|f_{n m}^{2,2,0}(t)\right| \leq N_{2} \sum_{n, m=1}^{\infty} \frac{1}{v_{n}^{2} \mu_{m}^{2}}=\frac{N_{2} p^{2} q^{2}}{36}
$$

Adding equality (5.12), (5.13), and (5.14), we note that the solution (3.1) satisfies equation (2.13). The solution (3.1) satisfies boundary conditions owing to properties of the functions $v_{n m}(x, y)$. Simple computations show that

$$
\begin{gathered}
\lim _{t \rightarrow 0} E_{\alpha, 1}\left(-\lambda_{n m}^{2 k} t^{\alpha}\right)=1, \\
\lim _{t \rightarrow 0} \frac{d}{d t} E_{\alpha, 1}\left(-\lambda_{n m}^{2 k} t^{\alpha}\right)=0, \\
\lim _{t \rightarrow 0} E_{\alpha, 2}\left(-\lambda_{n m}^{2 k} t^{\alpha}\right)=1, \\
\lim _{t \rightarrow 0} t \frac{d}{d t} E_{\alpha, 2}\left(-\lambda_{n m}^{2 k} t^{\alpha}\right)=0 .
\end{gathered}
$$

Consequently, $\lim _{t \rightarrow 0} u_{n m}(t)=\varphi_{n m}, \lim _{t \rightarrow 0} u_{n m}^{\prime}(t)=\psi_{n m}$. Hence, we conclude that the solution (3.1) satisfies initial conditions. Theorem 5.2 is proved.

\section{Conclusion}

In this paper, the initial-boundary value problem (2.13) for partial differential equations of higher order involving the Caputo fractional derivative is studied. Theorems on existence and uniqueness of a solution and its continuous dependence on the initial data and on the 
right-hand side of the equation are established. Of course, such type of results have been established for the initial-boundary value problem:

$$
\begin{aligned}
& (-1)^{k c} D_{0+}^{\alpha} u+\frac{\partial^{2 k} u}{\partial x^{2 k}}+\frac{\partial^{2 k} u}{\partial y^{2 k}}+u=f(x, y, t), \quad 0<x<p, 0<y<q, 0<t<T, \\
& \frac{\partial^{2 m+1} u(0, y, t)}{\partial x^{2 m}}=\frac{\partial^{2 m+1} u(p, y, t)}{\partial x^{2 m}}=0, \quad m=0,1, \ldots, k-1,0 \leq y \leq q, 0 \leq t \leq T, \\
& \frac{\partial^{2 m+1} u(x, 0, t)}{\partial y^{2 m}}=\frac{\partial^{2 m+1} u(x, q, t)}{\partial y^{2 m}}=0, \quad m=0,1, \ldots, k-1,0 \leq x \leq p, 0 \leq t \leq T, \\
& u(x, y, 0)=\varphi(x, y), \quad u_{t}(x, y, 0)=\psi(x, y), \quad 0 \leq x \leq p, 0 \leq y \leq q
\end{aligned}
$$

for partial differential equations of higher order with a fractional derivative of order $\alpha \in(1,2)$ in the sense of Caputo. Here, $k(k \geq 1)$ is a fixed positive integer number.

Moreover, applying the result of the papers [15, 23], the first order of accuracy difference schemes for the numerical solution of nonlocal boundary value problems (2.13) and (6.1) can be presented. Of course, the stability inequalities for the solution of these difference schemes have been established without any assumptions about the grid steps $\tau$ in $t$ and $h$ in the space variables.

\section{Acknowledgment}

The authors are grateful to Professor Valery Covachev (Sultan Qaboos University, Sultanate of Oman) for his insightful comments and suggestions.

\section{References}

[1] R. L. Bagley and P. J. Torvik, "A theoretical basis for the application of fractional calculus to viscoelasticity," Journal of Rheology, vol. 27, no. 3, pp. 201-210, 1983.

[2] G. Sorrentinos, "Fractional derivative linear models for describing the viscoelastic dynamic behavior of polymeric beams," in Proceedings of IMAS, Saint Louis, Mo, USA, 2006.

[3] G. Sorrentinos, "Analytic modeling and experimental identi?cation of viscoelastic mechanical systems," in Advances in Fractional Calculus, J. Sabatier, O. P. Agrawal, and J. A Tenreiro Machado, Eds., pp. 403-416, Springer, 2007.

[4] Fractals and Fractional Calculus in Continuum Mechanics, vol. 378 of CISM Courses and Lectures, Springer, New York, NY, USA, 1997.

[5] R. L. Magin, "Fractional calculus in bioengineering," Critical Reviews in Biomedical Engineering, vol. 32, no. 1, pp. 1-104, 2004.

[6] M. D. Ortigueira and J. A. Tenreiro Machado, "Special issue on Fractional signal processing and applications," Signal Processing, vol. 83, no. 11, pp. 2285-2286, 2003.

[7] B. M. Vinagre, I. Podlubny, A. Hernández, and V. Feliu, "Some approximations of fractional order operators used in control theory and applications," Fractional Calculus E Applied Analysis, vol. 3, no. 3, pp. 231-248, 2000.

[8] K. B. Oldham, "Fractional differential equations in electrochemistry," Advances in Engineering Software, vol. 41, no. 1, pp. 9-12, 2010.

[9] R. Metzler and J. Klafter, "Boundary value problems for fractional diffusion equations," Physica A, vol. 278, no. 1-2, pp. 107-125, 2000. 
[10] M. De la Sen, "Positivity and stability of the solutions of Caputo fractional linear time-invariant systems of any order with internal point delays," Abstract and Applied Analysis, vol. 2011, Article ID 161246, 25 pages, 2011.

[11] I. Podlubny, Fractional Differential Equations, vol. 198 of Mathematics in Science and Engineering, Academic Press, New York, NY, USA, 1999.

[12] S. G. Samko, A. A. Kilbas, and O. I. Marichev, Fractional Integrals and Derivatives, Gordon and Breach Science Publishers, London, UK, 1993.

[13] A. A. Kilbas, H. M. Srivastava, and J. J. Trujillo, Theory and Applications of Fractional Differential Equations, vol. 204 of North-Holland Mathematics Studies, Elsevier Science B.V., Amsterdam, The Nrtherlands, 2006.

[14] J.-L. Lavoie, T. J. Osler, and R. Tremblay, "Fractional derivatives and special functions," SIAM Review, vol. 18, no. 2, pp. 240-268, 1976.

[15] A. Ashyralyev, "A note on fractional derivatives and fractional powers of operators," Journal of Mathematical Analysis and Applications, vol. 357, no. 1, pp. 232-236, 2009.

[16] C. Yuan, "Two positive solutions for $(n-1,1)$-type semipositone integral boundary value problems for coupled systems of nonlinear fractional differential equations," Communications in Nonlinear Science and Numerical Simulation, vol. 17, no. 2, pp. 930-942, 2012.

[17] M. De la Sen, R. P. Agarwal, A. Ibeas, and S. Alonso-Quesada, "On the existence of equilibrium points, boundedness, oscillating behavior and positivity of a SVEIRS epidemic model under constant and impulsive vaccination," Advances in Difference Equations, vol. 2011, Article ID 748608, 32 pages, 2011.

[18] O. P. Agrawal, "Formulation of Euler-Lagrange equations for fractional variational problems," Journal of Mathematical Analysis and Applications, vol. 272, no. 1, pp. 368-379, 2002.

[19] R. W. Ibrahim and S. Momani, "On the existence and uniqueness of solutions of a class of fractional differential equations," Journal of Mathematical Analysis and Applications, vol. 334, no. 1, pp. 1-10, 2007.

[20] V. Lakshmikantham and A. S. Vatsala, "Basic theory of fractional differential equations," Nonlinear Analysis, vol. 69, no. 8, pp. 2677-2682, 2008.

[21] R. P. Agarwal, M. Benchohra, and S. Hamani, "Boundary value problems for fractional differential equations," Georgian Mathematical Journal, vol. 16, no. 3, pp. 401-411, 2009.

[22] A. Ashyralyev and B. Hicdurmaz, "A note on the fractional Schrödinger differential equations," Kybernetes, vol. 40, no. 5-6, pp. 736-750, 2011.

[23] A. Ashyralyev, F. Dal, and Z. Pınar, "A note on the fractional hyperbolic differential and difference equations," Applied Mathematics and Computation, vol. 217, no. 9, pp. 4654-4664, 2011.

[24] A. Ashyralyev and Z. Cakir, "On the numerical solution of fractional parabolic partial differential equations," AIP Conference Proceeding, vol. 1389, pp. 617-620, 2011.

[25] A. Ashyralyev, "Well-posedness of the Basset problem in spaces of smooth functions," Applied Mathematics Letters, vol. 24, no. 7, pp. 1176-1180, 2011.

[26] A. A. Kilbas and O. A. Repin, “Analogue of Tricomi's problem for partial differential equations containing diffussion equation of fractional order," in Proceedings of the International Russian-Bulgarian Symposium Mixed type equations and related problems of analysis and informatics, pp. 123-127, NalchikHaber, 2010.

[27] A. A. Nahushev, Elements of Fractional Calculus and Their Applications, Nalchik, Russia, 2010.

[28] A. V. Pshu, Boundary Value Problems for Partial Differential Equations of Fractional and Continual Order, Nalchik, Russia, 2005.

[29] N. A. Virchenko and V. Y. Ribak, Foundations of Fractional Integro-Differentiations, Kiev, Ukraine, 2007.

[30] M. M. Džrbašjan and A. B. Nersesjan, "Fractional derivatives and the Cauchy problem for differential equations of fractional order," Izvestija Akademii Nauk Armjanskŏ SSR, vol. 3, no. 1, pp. 3-28, 1968.

[31] R. P. Agarwal, M. Benchohra, and S. Hamani, "A survey on existence results for boundary value problems of nonlinear fractional differential equations and inclusions," Acta Applicandae Mathematicae, vol. 109, no. 3, pp. 973-1033, 2010.

[32] R. P. Agarwal, M. Belmekki, and M. Benchohra, "A survey on semilinear differential equations and inclusions involving Riemann-Liouville fractional derivative," Advances in Difference Equations, vol. 2009, Article ID 981728, 47 pages, 2009.

[33] R. P. Agarwal, B. de Andrade, and C. Cuevas, "On type of periodicity and ergodicity to a class of fractional order differential equations," Advances in Difference Equations, vol. 2010, Article ID 179750, 25 pages, 2010. 
[34] R. P. Agarwal, B. de Andrade, and C. Cuevas, "Weighted pseudo-almost periodic solutions of a class of semilinear fractional differential equations," Nonlinear Analysis, vol. 11, no. 5, pp. 3532-3554, 2010.

[35] D. Araya and C. Lizama, "Almost automorphic mild solutions to fractional differential equations," Nonlinear Analysis, vol. 69, no. 11, pp. 3692-3705, 2008.

[36] G. M. N'Guérékata, "A Cauchy problem for some fractional abstract differential equation with non local conditions," Nonlinear Analysis, vol. 70, no. 5, pp. 1873-1876, 2009.

[37] G. M. Mophou and G. M. N'Guérékata, "Mild solutions for semilinear fractional differential equations," Electronic Journal of Differential Equations, vol. 2009, no. 21, 9 pages, 2009.

[38] G. M. Mophou and G. M. N'Guérékata, "Existence of the mild solution for some fractional differential equations with nonlocal conditions," Semigroup Forum, vol. 79, no. 2, pp. 315-322, 2009.

[39] V. Lakshmikantham, "Theory of fractional functional differential equations," Nonlinear Analysis, vol. 69, no. 10, pp. 3337-3343, 2008.

[40] V. Lakshmikantham and J. V. Devi, "Theory of fractional differential equations in a Banach space," European Journal of Pure and Applied Mathematics, vol. 1, no. 1, pp. 38-45, 2008.

[41] V. Lakshmikantham and A. S. Vatsala, "Theory of fractional differential inequalities and applications," Communications in Applied Analysis, vol. 11, no. 3-4, pp. 395-402, 2007.

[42] A. S. Berdyshev, A. Cabada, and E. T. Karimov, “On a non-local boundary problem for a parabolichyperbolic equation involving a Riemann-Liouville fractional differential operator," Nonlinear Analysis, vol. 75, no. 6, pp. 3268-3273, 2011.

[43] R. Gorenflo, Y. F. Luchko, and S. R. Umarov, "On the Cauchy and multi-point problems for partial pseudo-differential equations of fractional order," Fractional Calculus E Applied Analysis, vol. 3, no. 3, pp. 249-275, 2000.

[44] B. Kadirkulov and K. H. Turmetov, "About one generalization of heat conductivity equation," Uzbek Mathematical Journal, no. 3, pp. 40-45, 2006 (Russian).

[45] D. Amanov, "Solvability of boundary value problems for equation of higher order with fractional derivatives," in Boundary Value Problems for Differential Equations, The Collection of Proceedings no. 17, pp. 204-209, Chernovtsi, Russia, 2008.

[46] D. Amanov, "Solvability of boundary value problems for higher order differential equation with fractional derivatives," in Problems of Camputations and Applied Mathemaitics, no. 121, pp. 55-62, Tashkent, Uzbekistan, 2009.

[47] M. M. Djrbashyan, Integral Transformations and Representatation of Functions in Complex Domain, Moscow, Russia, 1966. 


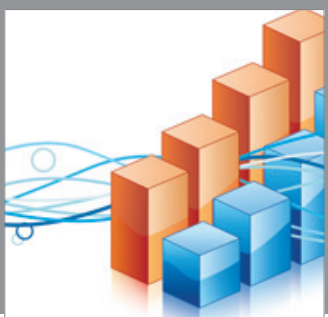

Advances in

Operations Research

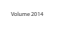

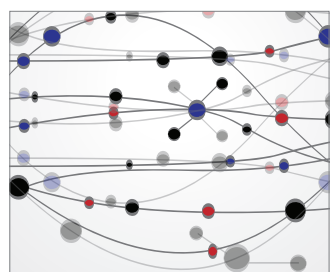

\section{The Scientific} World Journal
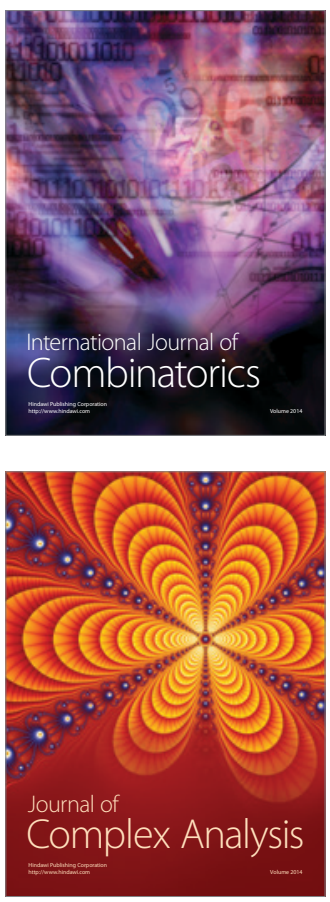

International Journal of

Mathematics and

Mathematical

Sciences
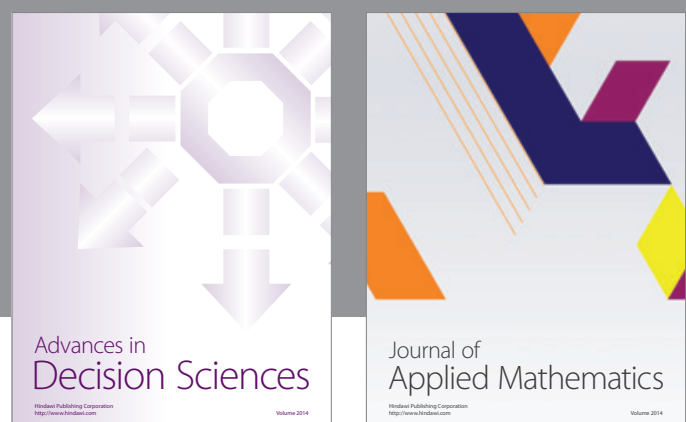

Journal of

Applied Mathematics
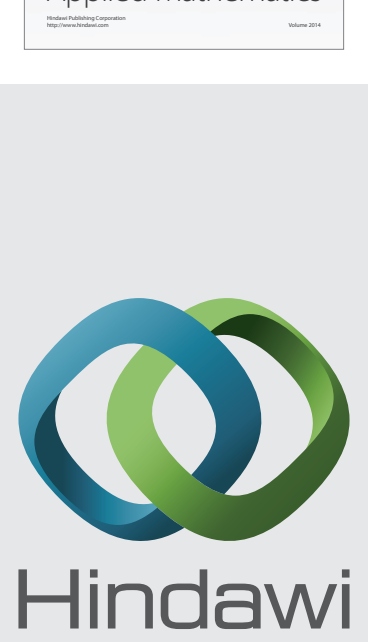

Submit your manuscripts at http://www.hindawi.com
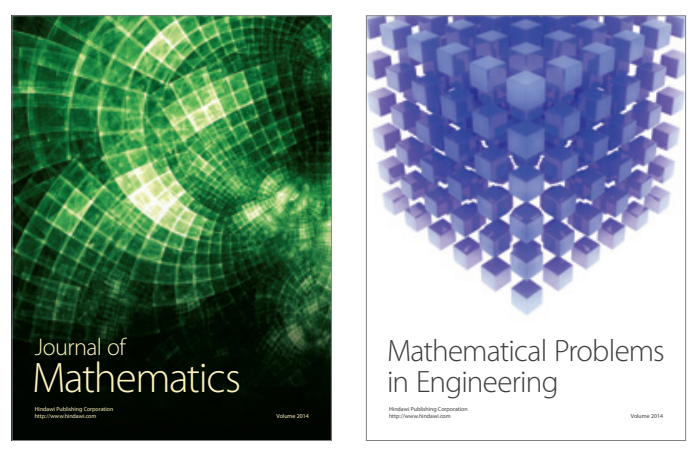

Mathematical Problems in Engineering
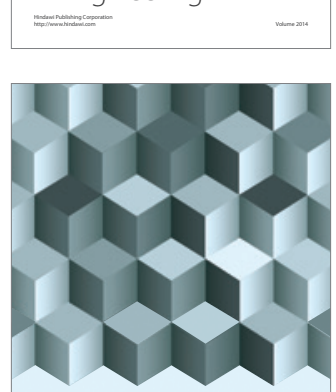

Journal of

Function Spaces
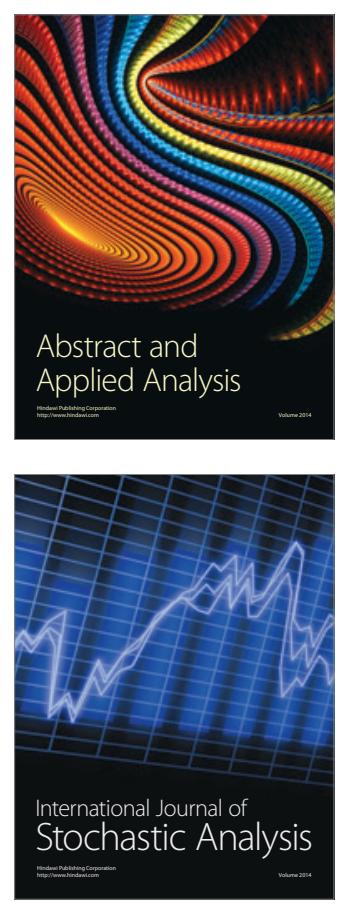

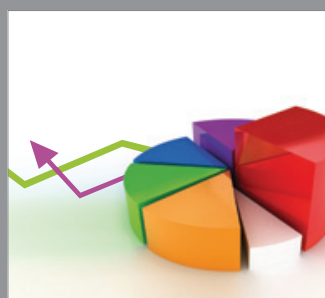

ournal of

Probability and Statistics

Promensencen
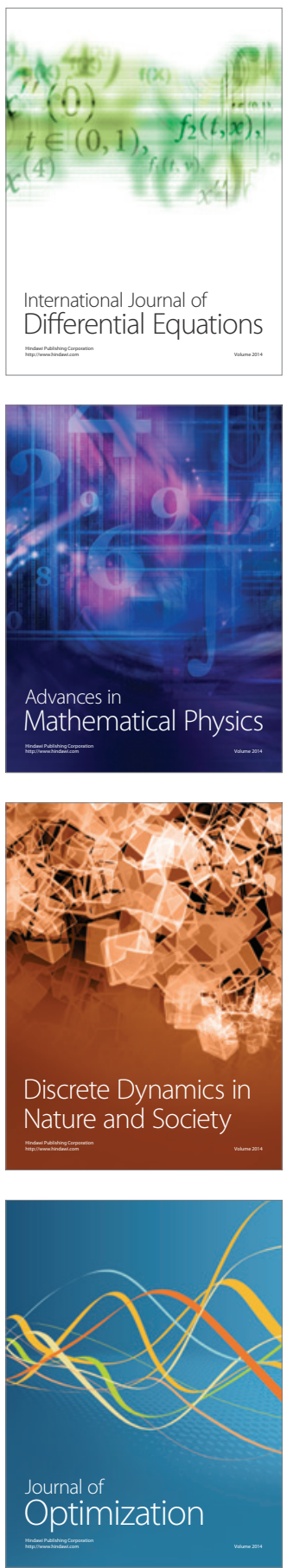